\title{
The polycystic ovary syndrome per se is not associated with increased chronic inflammation
}

Matthias Möhlig, Joachim Spranger, Martin Osterhoff, Michael Ristow, Andreas F H Pfeiffer, Thilo Schill ${ }^{1}$, Hans W Schlösser ${ }^{1}$, Georg Brabant ${ }^{2}$ and Christof Schöfl ${ }^{2}$

Department of Clinical Nutrition, German Institute of Human Nutrition, 14558 Potsdam-Rehbrücke, Germany and Department of Endocrinology, Diabetes and Nutrition, Charité-University Medicine Berlin, Campus Benjamin Franklin, 12200 Berlin, Germany, ${ }^{1}$ Department of Reproduction and Fertility, Hannover Medical School, 30623 Hannover, Germany and ${ }^{2}$ Department of Gastroenterology, Hepatology and Endocrinology, Hannover Medical School, 30623 Hannover, Germany

(Correspondence should be addressed to Christof Schöfl, Abteilung Gastroenterologie, Hepatologie und Endokrinologie, Medizinische Hochschule Hannover, Carl-Neuberg-Str. 1, 30625 Hannover, Germany; Email: schoefl.christof@mh-hannover.de)

\begin{abstract}
Objective: The syndrome of polycystic ovaries (PCOS) is a known risk factor for type 2 diabetes. It is not known, however, whether the increase in diabetes risk is related to endocrine abnormalities associated with PCOS such as hyperandrogenemia, or whether it is a consequence of the anthropometric or metabolic alterations frequently observed in PCOS women.

Design: Since markers of inflammation are supposed to predict type 2 diabetes, interleukin-6 (IL-6) and C-reactive protein (CRP) in combination with parameters of obesity, insulin resistance and hyperandrogenism were determined in 57 PCOS women and in 20 age-matched healthy controls. In addition, the C-174G IL- 6 promoter polymorphism was analyzed as a determinant in influencing IL-6, obesity, and androgen levels in women.

Results: Neither CRP nor IL-6 were significantly elevated in lean or obese PCOS women compared with age-matched lean or obese controls. In PCOS patients, variables of body composition (body mass index (BMI), waist to hip ratio, dual-energy X-ray-absorptiometry fat mass) and of insulin resistance were correlated with IL- 6 or CRP, while parameters of hyperandogenism were not. Multivariate linear regression analysis revealed that obesity is the dominant force, thus explaining $18 \%$ and $24 \%$ of the IL-6 or CRP levels, respectively, in PCOS women. No association of IL-6 or BMI to a certain genotype at $\mathrm{C}-174 \mathrm{G}$ could be demonstrated in 50 PCOS patients. The heterozygous GC genotype, however, was associated with lower androstendione levels. Metformin treatment of 9 obese, insulin-resistant PCOS patients over a period of 6 months caused a significant decrease in body weight, body fat mass and total testosterone, but showed no significant decline in IL-6 or CRP concentrations.

Conclusions: In PCOS women, plasma levels of IL-6 and CRP were not increased when compared with age- and BMI-matched controls. BMI was, however, the parameter most strongly related to IL-6 and CRP in PCOS; thus PCOS-related endocrine abnormalities do not appear to activate inflammatory parameters thereby enhancing the risk of diabetes. In PCOS, the type 2 diabetes risk may, therefore, be confined to those with obesity and/or metabolic alterations rather than affecting all women suffering from the syndrome.
\end{abstract}

European Journal of Endocrinology 150 525-532

\section{Introduction}

The polycystic ovary syndrome (PCOS), which is characterized by hyperandrogenism, chronic anovulation and infertility, is one of the most frequent endocrine disorders in women $(1-4)$. In addition to the reproductive abnormalities, a significant proportion of PCOS women suffer from obesity, insulin resistance and features of the metabolic syndrome $(1,2)$. Amelioration of metabolic abnormalities, especially those related to insulin resistance, by lifestyle or pharmacological intervention has been shown to improve hyperandrogenism and fertility (5-8). This indicates a close link between the reproductive and metabolic disturbances in PCOS.

In recent years, several studies have demonstrated a high risk for impaired glucose tolerance and type 2 diabetes mellitus in PCOS $(9-11)$. It has not yet been clarified whether this increase in risk is related to endocrine abnormalities associated with PCOS per se, such as hyperandrogenemia, or whether it is a consequence of the anthropometric or metabolic abnormalities frequently observed in PCOS women. In the latter case, type 2 diabetes risk would be confined to those women with anthropometric or metabolic alterations 
rather than affecting all women suffering from PCOS. This knowledge would be important for individual risk assessment and for strategies focusing on type 2 diabetes prevention in PCOS patients.

Markers of chronic subclinical inflammation such as C-reactive protein (CRP) or interleukin-6 (IL-6) have been shown to be independent predictors of risk for the development of type 2 diabetes (12-15). Consistently increased CRP levels have been reported in PCOS patients (16), supporting the hypothesis that PCOS increases diabetes risk by activating chronic inflammation. Circulating CRP and IL-6 concentrations are correlated to obesity as well as to insulin resistance (17-19), both of which are common findings in PCOS, and IL-6 expression in adipose tissue correlates with obesity (17). It is still not known whether these parameters of chronic inflammation are primary or secondary to obesity and/or insulin resistance especially since short term administration of IL- 6 in humans failed to impair insulin sensitivity (20). In addition, the $\mathrm{C}-174 \mathrm{G}$ polymorphism within the IL- 6 gene promoter region, which has been shown to modify promoter activity in vitro (21), has been linked to increased IL-6 levels and to hyperandrogenism in a small cohort of lean females (22). Thus, IL-6 may provide a link between anthropometric and metabolic alterations and hyperandrogenism in PCOS.

In order to investigate a possible relationship between IL-6, hyperandrogenemia and anthropometric or metabolic alterations on the one hand, and to gain further insight into the significance of PCOS related endocrine and metabolic abnormalities for the high risk of type 2 diabetes on the other hand, we measured both IL-6 and CRP as parameters of chronic inflammation and risk markers for type 2 diabetes, as well as parameters of obesity, insulin resistance and hyperandrogenism in 57 PCOS women and in 20 agematched healthy controls. The C-174G IL-6 promoter polymorphism was analyzed in 50 PCOS women.

\section{Subjects and methods}

\section{Subjects}

Fifty-seven women with PCOS participated in the study after informed consent was obtained. The diagnosis was based on clinical symptoms, i.e. oligo- or amenorrhea and hirsutism, and on laboratory findings, i.e. serum androgen levels (dehydroepiandrosterone sulfate (DHEAS), 17-hydroxy-progesterone (17-OH-progesterone), androstendione or testosterone) above the upper limit of normal for the respective assay and/or elevated luteinizing hormone/follicle-stimulating hormone (LH/ FSH) ratio. Cushing's syndrome, late-onset 21-hydroxylase deficiency, thyroid dysfunction, hyperprolactinemia or androgen secreting tumors were excluded. Two patients suffered from Hashimoto's thyroiditis. They were euthyroid under thyroid hormone
Table 1 Clinical and endocrine features of PCOS patients and healthy controls. The results are expressed as means \pm S.E.M.

\begin{tabular}{|c|c|c|c|}
\hline Variable & $\begin{array}{c}\text { Controls } \\
(n=20)\end{array}$ & $\begin{array}{c}\text { PCOS } \\
(n=57)\end{array}$ & $\boldsymbol{P}^{\mathrm{a}}$ \\
\hline Age (years) & $30.5 \pm 1.5$ & $28.9 \pm 0.7$ & 0.33 \\
\hline BMI $\left(\mathrm{kg} / \mathrm{m}^{2}\right)$ & $26.0 \pm 1.3$ & $30.3 \pm 1.0$ & 0.037 \\
\hline WHR & $0.79 \pm 0.01$ & $0.80 \pm 0.01$ & 0.37 \\
\hline Fasting glucose (mmol/l) & $5.0 \pm 0.2$ & $4.5 \pm 0.1$ & 0.009 \\
\hline Fasting insulin (pmol/l) & $56.9 \pm 6.7$ & $88.8 \pm 7.8$ & 0.012 \\
\hline HOMA (\%S) & $113.8 \pm 12.8$ & $87.1 \pm 7.0$ & 0.016 \\
\hline $\mathrm{LH}(\mathrm{mU} / \mathrm{ml})^{\prime}$ & $6.03 \pm 0.61$ & $9.01 \pm 0.70$ & 0.013 \\
\hline $\mathrm{LH} / \mathrm{FSH}$ & $0.97 \pm 0.09$ & $1.80 \pm 0.17$ & 0.002 \\
\hline $\mathrm{E}_{2}(\mathrm{pg} / \mathrm{ml})$ & $70.8 \pm 7.1$ & $68.9 \pm 12.5$ & 0.018 \\
\hline Progesterone (ng/ml) & $0.93 \pm 0.13$ & $1.45 \pm 0.44$ & 0.64 \\
\hline Testosterone (ng/ml) & $0.63 \pm 0.05$ & $0.93 \pm 0.05$ & 0.002 \\
\hline SHBG $(\mathrm{nmol} / \mathrm{l})$ & $72.0 \pm 6.6$ & $61.3 \pm 7.2$ & 0.043 \\
\hline Androstendione (ng/ml) & $1.33 \pm 0.16$ & $2.43 \pm 0.11$ & $<0.001$ \\
\hline 17-OH-progesterone (ng/ml) & $0.54 \pm 0.09$ & $0.84 \pm 0.08$ & 0.018 \\
\hline DHEAS $(\mu \mathrm{g} / \mathrm{ml})$ & $2.6 \pm 0.5$ & $2.9 \pm 0.1$ & 0.176 \\
\hline
\end{tabular}

${ }^{a}$ Non-parametric Mann-Whitney U test.

replacement therapy. The remaining patients were free of other diseases and were not under medication. Twenty age-matched healthy women were included as controls. Diabetes due to fasting glucose, smoking and contraceptive medication were exclusion criteria. The characteristics of PCOS women and controls are summarized in Table 1 . Nine obese and insulin-resistant PCOS women were re-evaluated after 6 months of treatment with $850 \mathrm{mg}$ metformin three times daily.

All of the women were studied within the first 10 days after onset of menstruation in the case of mild oligomenorrhea or at random in those suffering from severe oligo- or amenorrhea. Blood samples were taken in the morning subsequent to an overnight fast. Plasma was removed and stored at $-20{ }^{\circ} \mathrm{C}$ until analyzed. Insulin resistance was assessed by means of the homeostasis model (HOMA) using the computer program HOMA 2 provided by Dr Levy $(23,24)$. Body mass index (BMI) was calculated as body weight $(\mathrm{kg})$ divided by body height squared $\left(\mathrm{m}^{2}\right)$. Waist-to-hip ratio (WHR) was calculated as waist circumference divided by hip circumference.

\section{Assessment of body composition}

Body fat mass was assessed using whole-body scans by dual-energy X-ray-absorptiometry (DEXA, Lunar, Madison, WI, USA). The coefficient of variance was determined by repeated measurements and was $2.2 \%$ for total fat mass.

\section{Assays}

Blood glucose was measured by the glucosedehydrogenase method on the Cobas Mira laboratory system (Roche, Mannheim, Germany). Plasma insulin was measured by a commercial RIA (Pharmacia, Freiburg, 
Germany) with a lower limit of sensitivity of $<14 \mathrm{pmol} / \mathrm{l}$ and intra- and interassay coefficients of variation of $5.8 \%$ and $5.8 \%$ respectively. IL- 6 was determined by an ELISA (R\&D Systems, Minneapolis, MN, USA) according to the manufacturer's instructions. All samples were analyzed in one assay. The intraand interassay coefficients of variation were $12.6 \%$ and $9.3 \%$ respectively. CRP was measured with a turbidimetric test (Wako, Neuss, Germany) with the Cobas Mira laboratory system. Again, all samples were analyzed in one assay. The intra- and interassay coefficients of variation were $17 \%$ and $12 \%$ respectively. Serum 17-OH-progesterone was determined by RIA (Schering, Berlin, Germany) with a lower level of sensitivity of $2 \mathrm{ng} / 100 \mathrm{ml}$ and intra- and interassay coefficients of variation of $3.2 \%$ and $3.9 \%$ respectively. Serum LH and progesterone were measured by chemiluminescence immunoassays using kits obtained from Bayer Diagnostics (Fernwald, Germany). The intra- and interassay coefficients of variation were $2.9 \%$ and $2.4 \%$ for $\mathrm{LH}$ and $3.7 \%$ and $3.9 \%$ for progesterone, and the minimal detectable concentrations were $0.07 \mathrm{mU} / \mathrm{ml}(\mathrm{LH})$ and $0.15 \mathrm{ng} / \mathrm{ml}$ (progesterone). DHEAS was assayed by a chemiluminescence immunoassay purchased from Nichols Institute Diagnostics (Bad Nauheim, Germany) with a lower sensitivity of $10 \mathrm{ng} / \mathrm{ml}$ and intra- and interassay coefficients of variation of $7.1 \%$ and $9.0 \%$ respectively. Serum FSH was determined by an immunoradiometric assay (BioChem ImmunoSystems, Freiburg, Germany) with a lower sensitivity of $0.3 \mathrm{mU} / \mathrm{ml}$ and inter- and intra-assay coefficients of variation of $2 \%$ and $3.1 \%$ respectively. Estradiol $\left(E_{2}\right)$, testosterone and sex hormone binding globulin (SHBG) were measured by RIA obtained from DSL (Sinsheim, Germany) with lower detectable concentrations of $2.2 \mathrm{pg} / \mathrm{ml}, 0.08 \mathrm{ng} / \mathrm{ml}$, and $0.4 \mu \mathrm{g} / \mathrm{ml}$ respectively. The respective inter- and intra-assay coefficients of variation were $2.2 \%$ and $2.7 \%$ for $\mathrm{E}_{2}, 9.6 \%$ and $8.6 \%$ for testosterone, and $2.2 \%$ and $4.4 \%$ for SHBG. Androstendione was determined by RIA (Coulter Immunotech, Marseille, France) with a lower sensitivity of $4 \mathrm{ng} / 100 \mathrm{ml}$ and inter- and intra-assay coefficients of variation of $5.6 \%$ and $6 \%$ respectively.

\section{DNA extraction and genotype analysis}

DNA was extracted from blood cells using Magnasep magnetic beads (Agowa, Berlin, Germany). PCR was performed with the upper primer 5'-TAG CCT GTT AAT CTG GTC ACT G and the lower primer $5^{\prime}$-TAA ATC TTT GTT GGA GGG TG at $64^{\circ} \mathrm{C}$ annealing temperature $\left(2.5 \mathrm{mmol} / \mathrm{l} \mathrm{MgCl}_{2}\right)$. The single nucleotide polymorphism (SNP) diagnostic was performed by elongating the primer 5'- AAT GTG ACG TCC TTT AGC AT using SNuPE and following instructions and recommendations for purification by the manufacturer (Amersham, Piscataway, NJ, USA). Detection was performed on a MegaBACE 1000 (Molecular Dynamics,
Sunnyvale, CA, USA) using MegaBACE SNP profiler 1.0 software.

\section{Statistics}

Statistical analyses were performed with SPSS software (version 8.0, SPSS Inc., Chicago, IL, USA). Mean values are reported \pm standard error of the mean (S.E.M.). Significance was considered at two-tailed $\alpha<0.05$. The non-parametric Mann-Whitney $U$ test in the case of two groups or the Kruskal-Wallis test for comparison of three or more groups were used to analyze differences in skewed continuous variables. A paired Wilcoxon test was performed to compare characteristics before and during metformin treatment. Pearson correlation coefficients were used to test the correlation between variables. Linear regression analysis was performed within the PCOS cohort with In IL-6 or In CRP as the dependent variables. HOMA and BMI values were In-transformed to achieve normal distribution and were fitted stepwise into the model.

\section{Results}

The characteristics of the PCOS patients are summarized in Table 1. As anticipated in PCOS testosterone, $\mathrm{LH} / \mathrm{FSH}$ ratio, androstendione and 17-OH-progesterone were significantly increased. Furthermore, women suffering from PCOS had a higher BMI and were more insulin resistant than the age-matched healthy controls.

Plasma IL-6 and CRP concentrations were not found to be significantly different between PCOS and healthy control women (Fig. 1). Since both parameters are known to correlate with BMI, and because BMI showed a difference between PCOS and controls, we dichotomized our cohort at a BMI of $25 \mathrm{~kg} / \mathrm{m}^{2}$ to obtain a homogenous BMI distribution between PCOS women and controls (Table 2). The IL-6 and CRP levels were significantly higher in the groups with BMI $>25 \mathrm{~kg} / \mathrm{m}^{2}$ both in PCOS patients and in controls (Fig. 2). However, between BMI-matched PCOS and controls, neither IL- 6 nor CRP were significantly different despite higher androgen levels in the PCOS patients (Table 2, Fig. 2). Therefore, we could find no evidence for increased plasma IL- 6 or CRP levels in women suffering from PCOS.

Pearson correlations were calculated in order to define parameters associated with plasma levels of IL6 and CRP in PCOS. There was a highly significant positive correlation between plasma levels of IL- 6 and CRP $(r=0701 ; P<0.001)$ suggesting a close link between the two risk markers. As depicted in Fig. 3, IL-6 and CRP significantly correlated with parameters of obesity and insulin resistance (BMI, WHR, DEXA fat mass and HOMA). There was, however, no correlation of IL-6 or CRP to parameters of PCOS such as 

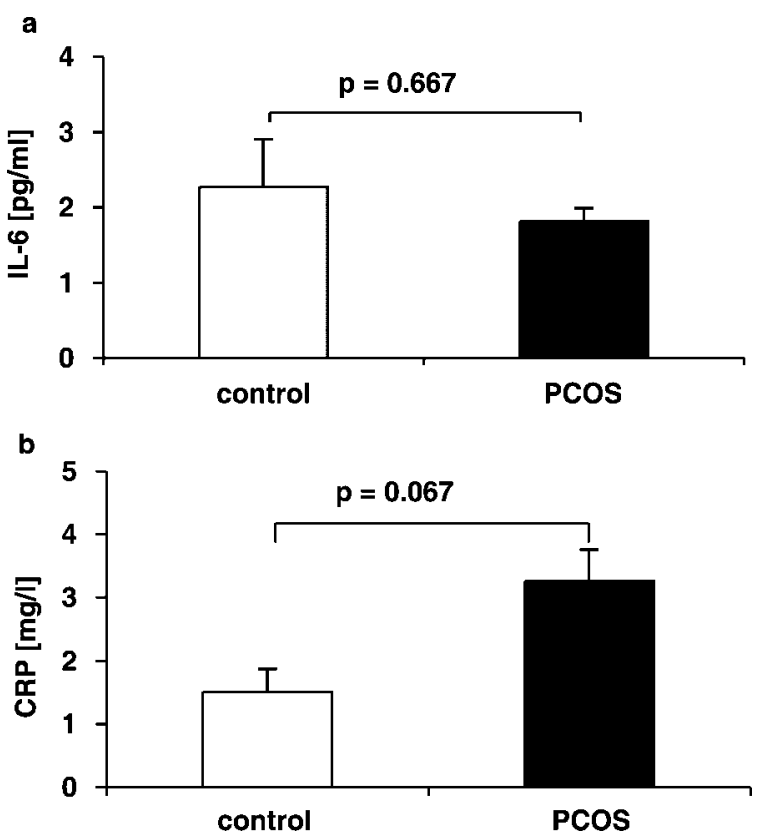

Figure 1 (a) IL-6 and (b) CRP plasma concentrations in 57 PCOS patients and 20 age-matched healthy controls. Results are expressed as means \pm S.E.M.

testosterone, androstendione, 17-OH-progesterone, DHEAS, LH or the LH/FSH ratio (data not shown). This indicates that obesity and metabolic alterations rather than endocrine changes associated with PCOS have an impact on markers of chronic inflammation in PCOS women.

Linear regression models were calculated in order to further investigate whether obesity (BMI) and/or insulin resistance (HOMA) determine markers of chronic inflammation in PCOS. Insulin resistance (HOMA) lost its significant association to IL-6 and CRP after inclusion of BMI into the model, while BMI remained significantly linked to both IL-6 and CRP. In the case of IL- 6 the model including HOMA and BMI showed an adjusted $\mathrm{R}^{2}$ of 0.18 . The beta coefficient for BMI was $1.28(P=0.02)$ and $-0.04 \quad(P=0.9)$ for HOMA. Regarding the model with CRP as the dependent variable, the adjusted $\mathrm{R}^{2}$ was 0.24 with a beta coefficient of $2.57(P=0.01)$ for BMI and of -0.13 $(P=0.76)$ for HOMA. Therefore, obesity appears to be the dominant force determining IL-6 and CRP values in PCOS women. Both models for BMI alone, however, explained only a small part of the variability of plasma IL-6 (18\%) and CRP (24\%). Therefore, additional factors controlling IL-6 and CRP concentrations must be assumed. These parameters, however, are different from the classical PCOS-associated endocrine factors such as hyperandrogenemia.

The IL-6 promoter polymorphism C-174G has been associated with altered IL-6 levels, obesity and hyperandrogenemia $(22,25-27)$. In a cohort of 50 PCOS women no association of IL- 6 levels or markers of obesity to a certain genotype at $\mathrm{C}-174 \mathrm{G}$ could be demonstrated (Table 3 ). This argues against a major impact of the C-174G IL-6 promoter polymorphism on IL-6 levels and obesity in PCOS patients. The heterozygous GC genotype was associated with statistically significant lower androstendione levels and with a tendency to lower testosterone concentrations (Table 3). There was, however, no association to either the $-174 \mathrm{G}$ or C allele.

Nine obese and insulin-resistant PCOS patients were treated with metformin. After 6 months therapy the women became less obese (BMI: $29.6 \pm 1.0 \mathrm{~kg} / \mathrm{m}^{2}$ vs $31.6 \pm 1.25 \mathrm{~kg} / \mathrm{m}^{2}, \quad P=0.025$; DEXA fat (total): $34376 \pm 1964 \mathrm{~g}$ vs $39444 \pm 2542 \mathrm{~g}, P=0.028)$ and testosterone levels significantly decreased (0.75 \pm $0.70 \mathrm{ng} / \mathrm{ml}$ vs $1.05 \pm 0.13 \mathrm{ng} / \mathrm{ml}, P=0.032)$. Insulin sensitivity improved, but the increase in HOMA\% (S) failed to reach significance $(83 \pm 8 \%$ (S) vs $69 \pm 9 \%$ $(\mathrm{S}) ; \mathrm{P}=0.14)$. IL-6 concentrations remained largely unchanged $(1.72 \pm 0.30 \mathrm{pg} / \mathrm{ml}$ vs $1.91 \pm 0.31 \mathrm{pg} / \mathrm{ml}$; $P=0.515)$ and CRP levels tended to drop after 6 months of metformin treatment, although the change was not significant $(1.92 \pm 0.29 \mathrm{mg} / \mathrm{l}$ vs $3.34 \pm 0.82 \mathrm{mg} / \mathrm{l} ; P=0.086)$.

Table 2 Clinical and endocrine features of PCOS patients and healthy controls dichotomized by BMI. The results are expressed as means \pm S.E.M.

\begin{tabular}{|c|c|c|c|c|}
\hline \multirow[b]{2}{*}{ Variable } & \multicolumn{2}{|c|}{ BMI < $25 \mathrm{~kg} / \mathrm{m}^{2}$} & \multicolumn{2}{|c|}{$\mathrm{BMI} \geq 25 \mathrm{~kg} / \mathrm{m}^{2}$} \\
\hline & Controls $(n=11)$ & $\operatorname{PCOS}(n=16)$ & Controls $(n=9)$ & $\operatorname{PCOS}(n=41)$ \\
\hline Age (years) & $29.4 \pm 1.9$ & $28.8 \pm 1.5$ & $32.0 \pm 2.3$ & $28.9 \pm 0.8$ \\
\hline $\mathrm{BMI}\left(\mathrm{kg} / \mathrm{m}^{2}\right)$ & $21.6 \pm 0.5$ & $21.5 \pm 0.5$ & $31.3 \pm 1.2$ & $33.7 \pm 1.0$ \\
\hline WHR & $0.77 \pm 0.01$ & $0.75 \pm 0.01$ & $0.82 \pm 0.03$ & $0.83 \pm 0.01$ \\
\hline HOMA (\%S) & $133.6 \pm 19.3$ & $132.6 \pm 15.1$ & $89.4 \pm 12.5$ & $69.3 \pm 5.9$ \\
\hline Testosterone (ng/ml) & $0.56 \pm 0.06$ & $0.73 \pm 0.07$ & $0.71 \pm 0.07$ & $1.00 \pm 0.06^{*}$ \\
\hline Androstendione (ng/ml) & $0.99 \pm 0.11$ & $2.31 \pm 0.20^{* \star *}$ & $1.53 \pm 0.22$ & $2.48 \pm 0.13^{\star \star}$ \\
\hline 17-OH-progesterone (ng/ml) & $0.44 \pm 0.05$ & $0.77 \pm 0.11^{\star}$ & $0.61 \pm 0.15$ & $0.86 \pm 0.11$ \\
\hline DHEAS $(\mu \mathrm{g} / \mathrm{ml})$ & $2.43 \pm 0.43$ & $2.84 \pm 0.35$ & $2.70 \pm 0.8$ & $2.88 \pm 0.22$ \\
\hline $\mathrm{LH}(\mathrm{mU} / \mathrm{ml})$ & $5.66 \pm 0.65$ & $10.05 \pm 1.55^{\star}$ & $6.02 \pm 1.15$ & $8.60 \pm 0.78$ \\
\hline $\mathrm{LH} / \mathrm{FSH}$ & $0.98 \pm 0.14$ & $1.85 \pm 0.29^{*}$ & $0.95 \pm 0.14$ & $1.77 \pm 0.21 *$ \\
\hline
\end{tabular}

$\star P<0.05 ; * \star P<0.01 ; * \star \star P<0.001$ PCOS compared with controls (non-parametric Mann-Whitney U test). 

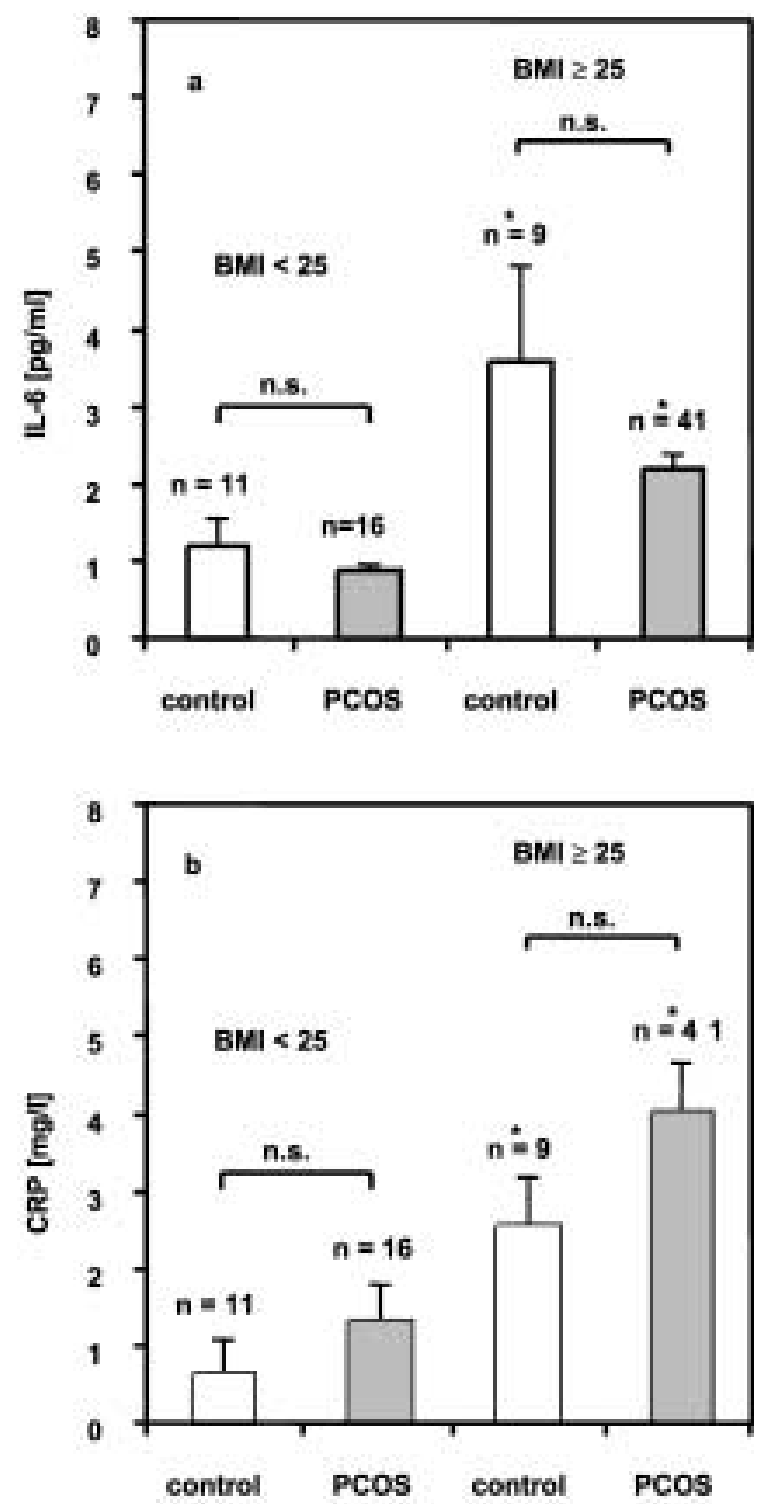

Figure 2 (a) IL-6 and (b) CRP plasma concentrations in PCOS patients and age-matched healthy controls dichotomized by BMI. Results are expressed as means \pm S.E.M. ${ }^{*} P<0.05$ vs the respective control or PCOS group with $\mathrm{BMI}<25 \mathrm{~kg} / \mathrm{m}^{2}$.

\section{Discussion}

PCOS is one of the most frequent endocrine disorders in women, with an estimated prevalence of $5-10 \%$ of the reproductive-age female population $(1-4)$. PCOS is a complex and heterogenous disorder and there is a debate as to whether it represents a single or several disorders with a common phenotype. A substantial subgroup of PCOS patients are obese and/or insulin resistant, and in these individuals hyperinsulinemia may play a major pathogenetic role in PCOS-associated hyperandrogenemia indicating a close link between the reproductive and metabolic disturbances $(1,2)$. Several studies suggested an increased risk for developing impaired glucose tolerance and type 2 diabetes mellitus in patients with PCOS $(9-11)$. It is, however, not known whether this increase in risk is related to endocrine abnormalities associated with PCOS per se, such as hyperandrogenemia, or whether it is a consequence of the anthropometric or metabolic alterations observed in some, but not in all, women with PCOS.

The development of type 2 diabetes has been linked to chronic inflammation in recent years and markers of activated inflammatory processes like IL-6 and CRP have been shown to predict the risk of type 2 diabetes (12-15). In our cohort of 57 PCOS women and 20 healthy age-matched controls no significant differences in plasma IL-6 or CRP levels could be demonstrated. This was also the case after the cohort was dichotomized at a BMI of $25 \mathrm{~kg} / \mathrm{m}^{2}$ to obtain a comparable mean BMI in PCOS patients and controls. This is in line with a report demonstrating the same IL-6 levels in 85 patients with hyperandrogenism and in 25 healthy controls (22), but is in contrast to another study describing elevated CRP levels in a cohort of 17 PCOS women compared with 15 controls (16). Our data, however, do not support the hypothesis that PCOS per se activates low grade chronic inflammation thereby increasing a priori the risk for the development of type 2 diabetes mellitus. This view is further supported by the fact that none of the endocrine markers of PCOS correlated with plasma IL-6 or CRP concentrations.

Plasma IL-6 and CRP levels were significantly increased in both obese PCOS and obese control women as compared with their lean counterparts, and both parameters correlated with markers of obesity and insulin resistance in the PCOS cohort. Linear regression models, however, revealed that $\mathrm{BMI}$ rather than insulin resistance as indicated by HOMA is the dominant parameter determining IL- 6 and CRP values in PCOS, which is consistent with a recent report from a cohort of premenopausal women (28). Since the models explain less than $30 \%$ of the variability of plasma IL-6 and CRP, additional factors controlling IL6 and CRP concentrations in PCOS must be assumed, which are, however, distinct from PCOS-associated endocrine factors such as hyperandrogenemia because none of the parameters of hyperandrogenism correlated with IL-6 or CRP.

One such factor might be the $\mathrm{C}-174 \mathrm{G}$ polymorphism within the IL- 6 gene promoter region, which has been suggested to modify promoter activity in vitro (21). The C-174G polymorphism has also been associated in some but not all studies with increased IL-6 levels, insulin resistance, resting energy expenditure, obesity and hyperandrogenism $(22,25,26,29,30)$. In our cohort of 50 PCOS patients, neither IL-6 levels nor markers of obesity were linked to a certain genotype at C-174G, which argues against a major impact of the C-174G IL-6 promoter polymorphism on IL-6 levels and obesity in PCOS patients. The heterozygous GC genotype, 

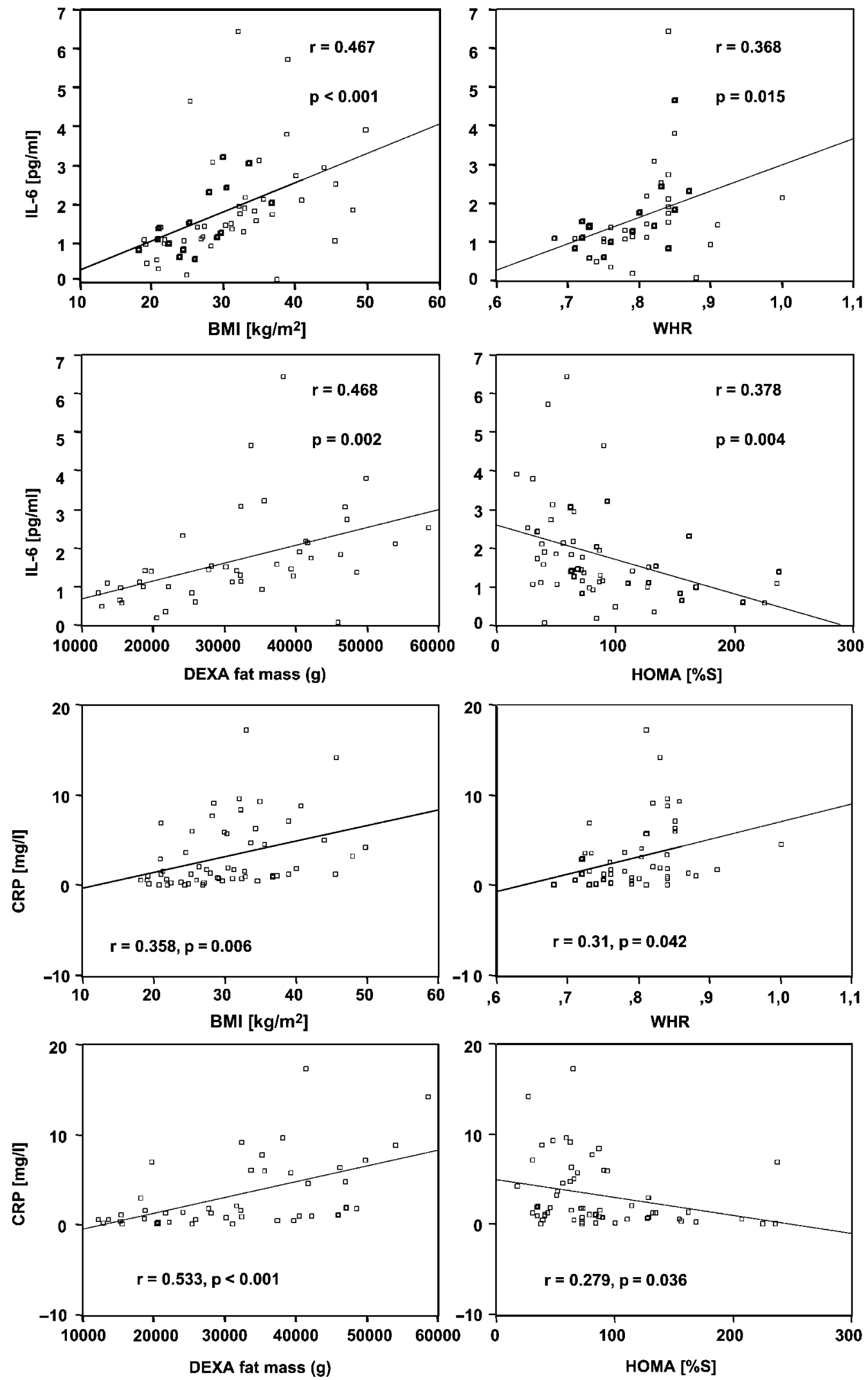

Figure 3 Pearson correlations between IL- 6 or CRP and BMI, WHR, DEXA fat mass and HOMA. 
Table 3 Clinical and endocrine features of 50 PCOS patients carrying different genotypes at the IL-6 promoter polymorphism C-174G. The results are expressed as means \pm S.E.M.

\begin{tabular}{lcccc}
\hline & \multicolumn{3}{c}{ Genotype } \\
\cline { 2 - 4 } Variable & GG $(n=19)$ & GC $(n=21)$ & CC $(n=10)$ & $31.1 \pm 1.3$ \\
\hline Age (years) & $27.7 \pm 1.3$ & $28.9 \pm 1.0$ & 0.21 \\
BMl $\left(\mathrm{kg} / \mathrm{m}^{2}\right)$ & $28.4 \pm 1.5$ & $29.5 \pm 1.3$ & $28.1 \pm 3.1$ & 0.63 \\
HOMA $(\% \mathrm{~S})$ & $87.5 \pm 13.2$ & $92.5 \pm 12.0$ & $95.6 \pm 17.4$ & 0.81 \\
Testosterone $(\mathrm{ng} / \mathrm{ml})$ & $1.1 \pm 0.1$ & $0.8 \pm 0.1$ & $1.0 \pm 0.2$ & 0.16 \\
Androstendione $(\mathrm{ng} / \mathrm{ml})$ & $2.69 \pm 0.19$ & $1.99 \pm 0.69$ & $2.9 \pm 0.22$ & 0.004 \\
DHEAS $(\mu \mathrm{g} / \mathrm{ml})$ & $3.30 \pm 0.38$ & $2.67 \pm 0.28$ & $0.70 \pm 0.36$ & 0.62 \\
17-OH-progesterone $(\mathrm{ng} / \mathrm{ml})$ & $1.0 \pm 0.1$ & $0.8 \pm 0.2$ & 0.1 & 0.09 \\
IL-6 $(\mathrm{pg} / \mathrm{ml})$ & $1.50 \pm 0.22$ & $2.0 \pm 0.32$ & $1.50 \pm 0.30$ & 0.49 \\
CRP $(\mathrm{mg} / \mathrm{l})$ & $4.10 \pm 1.10$ & $3.80 \pm 0.90$ & $1.20 \pm 0.40$ & 0.09 \\
\hline
\end{tabular}

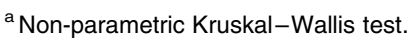

however, was associated with lower androstendione and a tendency to lower testosterone levels rather than to hyperandrogenemia. This is in contrast to the findings reported from 25 healthy women, whereby a significant association of the $-174 \mathrm{G}$ allele with elevated $17-\mathrm{OH}-$ progesterone levels but no links to androstendione or testosterone was shown (22). Whether this discrepancy can be explained by differences between PCOS patients and normal healthy women cannot be ascertained because of the relatively small sizes of both study cohorts. Since there were no differences in the circulating IL-6 levels in our cohort of PCOS patients, local IL-6 production, which is known to be expressed in the ovaries $(31,32)$, might be affected by the GC genotype by other, as yet unknown, mechanisms, which in turn might lead to decreased ovarian androgen secretion. Further studies, however, are required to elucidate the precise role of the C-174G IL-6 promotor polymorphism on androgen production in PCOS.

Metformin treatment in patients with PCOS resulted in a significant weight loss and reduction of total fat mass and caused a decrease of total testosterone levels as reported (6). Plasma IL-6 and CRP, however, did not change significantly although the decrease in CRP concentrations just failed to reach significance. Again, this does not support a direct link between hyperandrogenemia and low grade chronic inflammatory processes in PCOS. A decrease in BMI by $5.2 \mathrm{~kg} / \mathrm{m}^{2}$ has been shown to significantly reduce IL- 6 and CRP levels by about 33\% (33). In the linear regression models BMI, which was proposed as the dominant force, determined only 18 to $24 \%$ of the variability of IL- 6 or CRP respectively. Therefore, one explanation for the lack of significant changes in plasma IL-6 and CRP, despite significant weight loss, could be that the extent to which BMI and fat mass improved was not sufficient enough to cause a significant decrease in the inflammatory parameters. Furthermore, metformin treatment does not appear to exert additional or favorable effects on IL- 6 and CRP levels.

In summary, in the group of PCOS women, plasma levels of IL-6 and CRP were not increased when compared with age- and BMI-matched controls. BMI was the parameter most strongly associated with IL-6 and CRP in PCOS. Thus, PCOS-related endocrine abnormalities do not appear to further enhance the type 2 diabetes risk level, which is primarily set by obesity and metabolic alterations. The type 2 diabetes risk may therefore be confined to those with obesity and/or metabolic alterations rather than affecting all women suffering from the syndrome. Further prospective studies, however, are needed to prove this conclusion which has been based on risk markers that can only serve as surrogate parameters.

\section{Acknowledgements}

We thank Katrin Sprengel, Stefanie Nedel and Bärbel Thon for excellent technical assistance, and A Coughlan for linguistic help.

\section{References}

1 Dunaif A. Insulin resistance and the polycystic ovary syndrome: mechanism and implications for pathogenesis. Endocrine Reviews $199718774-800$.

2 Franks S. Polycystic ovary syndrome. New England Journal of Medicine $1995333853-861$.

3 Asuncion M, Calvo RM, San Millan JL, Sancho J, Avila S \& Escobar-Morreale HF. A prospective study of the prevalence of the polycystic ovary syndrome in unselected Caucasian women from Spain. Journal of Clinical Endocrinology and Metabolism $2000852434-2438$.

4 Knochenhauer ES, Key TJ, Kahsar-Miller M, Waggoner W, Boots LR \& Azziz R. Prevalence of the polycystic ovary syndrome in unselected black and white women of the southeastern United States: a prospective study. Journal of Clinical Endocrinology and Metabolism $1998 \mathbf{8 3} 3078-3082$.

5 Pasquali R \& Gamberini A. Treatment of the polycystic ovary syndrome with lifestyle intervention. Curr Opin Endocrinol Diabetes $20029459-468$.

6 Harborne L, Fleming R, Lyall H, Norman J \& Sattar N. Descriptive review of the evidence for the use of metformin in polycystic ovary syndrome. Lancet $2003 \mathbf{3 6 1} 1894-1901$.

7 Haas DA, Carr BR \& Attia GR. Effects of metformin on body mass index, menstrual cyclicity, and ovulation induction in women 
with polycystic ovary syndrome. Fertility and Sterility $2003 \mathbf{7 9}$ 469-481.

8 Costello MF \& Eden JA. A systematic review of the reproductive system effects of metformin in patients with polycystic ovary syndrome. Fertility and Sterility $2003 \mathbf{7 9} 1-13$.

9 Legro RS. Diabetes prevalence and risk factors in polycystic ovary syndrome. Current Opinion in Endocrinology and Diabetes $20029451-458$.

10 Legro RS, Kunselman AR, Dodson WC \& Dunaif A. Prevalence and predictors of risk for type 2 diabetes mellitus and impaired glucose tolerance in polycystic ovary syndrome: a prospective, controlled study in 254 affected women. Journal of Clinical Endocrinology and Metabolism 199984 165-169.

11 Ehrmann DA, Barnes RB, Rosenfield RL, Cavaghan MK \& Imperial J. Prevalence of impaired glucose tolerance and diabetes in women with polycystic ovary syndrome. Diabetes Care 199922 141-146.

12 Thorand B, Lowel H, Schneider A, Kolb H, Meisinger C, Frohlich M et al. C-reactive protein as a predictor for incident diabetes mellitus among middle-aged men: results from the MONICA Augsburg Cohort Study, 1984-1998. Archives of Internal Medicine 2003163 93-99.

13 Spranger J, Kroke A, Möhlig M, Hoffmann K, Bergmann MM, Ristow $\mathrm{M}$ et al. Inflammatory cytokines and the risk to develop type 2 diabetes: results of the prospective population-based European Prospective Investigation into Cancer and Nutrition (EPIC) - Potsdam Study. Diabetes 200352 812-817.

14 Pradhan AD, Manson JE, Rifai N, Buring JE \& Ridker PM. C-reactive protein, interleukin 6 , and risk of developing type 2 diabetes mellitus. Journal of the American Medical Association 2001286 $327-334$.

15 Freeman DJ, Norrie J, Caslake MJ, Gaw A, Ford I, Lowe GD et al. C-reactive protein is an independent predictor of risk for the development of diabetes in the West of Scotland Coronary Prevention Study. Diabetes 200251 1596-1600.

16 Kelly CC, Lyall H, Petrie JR, Gould GW, Connell JM \& Sattar N. Low grade chronic inflammation in women with polycystic ovarian syndrome. Journal of Clinical Endocrinology and Metabolism $2001862453-2455$.

17 Engeli S, Feldpausch M, Gorzelniak K, Hartwig F, Heintze U, Janke J et al. Association between adiponectin and mediators of inflammation in obese women. Diabetes 200352 942-947.

18 Festa A, D’Agostino R Jr, Howard G, Mykkanen L, Tracy RP \& Haffner SM. Chronic subclinical inflammation as part of the insulin resistance syndrome: the Insulin Resistance Atherosclerosis Study (IRAS). Circulation 2000102 42-47.

19 Fernandez-Real JM, Vayreda M, Richart C, Gutierrez C, Broch M, Vendrell J et al. Circulating interleukin-6 levels, blood pressure, and insulin sensitivity in apparently healthy men and women. Journal of Clinical Endocrinology and Metabolism 200186 1154-1159.

20 Steensberg A, Fischer CP, Sacchetti M, Keller C, Osada T, Schjerling $\mathrm{P}$ et al. Acute interleukin-6 administration does not impair muscle glucose uptake or whole-body glucose disposal in healthy humans. Journal of Physiology $2003 \mathbf{5 4 8} 631-638$.

21 Fishman D, Faulds G, Jeffery R, Mohamed-Ali V, Yudkin JS, Humphries $\mathrm{S}$ et al. The effect of novel polymorphisms in the interleukin-6 (IL-6) gene on IL-6 transcription and plasma IL-6 levels, and an association with systemic-onset juvenile chronic arthritis. Journal of Clinical Investigation $1998 \mathbf{1 0 2}$ $1369-1376$.

22 Villuendas G, San Millan JL, Sancho J \& Escobar-Morreale HF. The $-597 \mathrm{G} \rightarrow \mathrm{A}$ and $-174 \mathrm{G} \rightarrow \mathrm{C}$ polymorphisms in the promoter of the IL-6 gene are associated with hyperandrogenism. Journal of Clinical Endocrinology and Metabolism 200287 1134-1141.

23 Rudenski AS, Matthews DR, Levy JC \& Turner RC. Understanding 'insulin resistance': both glucose resistance and insulin resistance are required to model human diabetes. Metabolism $1991 \mathbf{4 0}$ 908-917.

24 Matthews DR, Hosker JP, Rudenski AS, Naylor BA, Treacher DF \& Turner RC. Homeostasis model assessment: insulin resistance and beta-cell function from fasting plasma glucose and insulin concentrations in man. Diabetologia 198528 1891-1892.

25 Jones KG, Brull DJ, Brown LC, Sian M, Greenhalgh RM, Humphries SE et al. Interleukin-6 (IL-6) and the prognosis of abdominal aortic aneurysms. Circulation 2001103 2260-2265.

26 Hulkkonen J, Pertovaara M, Antonen J, Pasternack A \& Hurme M. Elevated interleukin- 6 plasma levels are regulated by the promoter region polymorphism of the IL-6 gene in primary Sjogren's syndrome and correlate with the clinical manifestations of the disease. Rheumatology 200140 656-661.

27 Berthier MT, Paradis AM, Tchernof A, Bergeron J, Prud'homme D, Despres JP et al. The interleukin 6-174G/C polymorphism is associated with indices of obesity in men. Journal of Human Genetics $2003 \mathbf{4 8} 14-19$.

28 Escobar-Morreale HF, Villuendas G, Botella-Carretero JI, Sancho J \& San Millan JL. Obesity, and not insulin resistance, is the major determinant of serum inflammatory cardiovascular risk markers in pre-menopausal women. Diabetologia 200346 625-633.

29 Kubaszek A, Pihlajamaki J, Punnonen K, Karhapaa P, Vauhkonen I \& Laakso M. The C-174G promoter polymorphism of the IL- 6 gene affects energy expenditure and insulin sensitivity. Diabetes 200352 $558-561$.

30 Fernandez-Real JM, Broch M, Vendrell J, Gutierrez C, Casamitjana R, Pugeat M et al. Interleukin-6 gene polymorphism and insulin sensitivity. Diabetes 200049 517-520.

31 Buscher U, Chen FC, Kentenich H \& Schmiady H. Cytokines in the follicular fluid of stimulated and non-stimulated human ovaries; is ovulation a suppressed inflammatory reaction? Human Reproduction $1999 \mathbf{1 4} 162-166$.

32 Papanicolaou DA \& Vgontzas AN. Interleukin-6: the endocrine cytokine. Journal of Clinical Endocrinology and Metabolism 2000 $851331-1333$.

33 Esposito K, Pontillo A, Di Palo C, Giugliano G, Masella M, Marfella $\mathrm{R}$ et al. Effect of weight loss and lifestyle changes on vascular inflammatory markers in obese women: a randomized trial. Journal of the American Medical Association $2003 \mathbf{2 8 9}$ 1799-1804.

Received 4 November 2003

Accepted 20 January 2004 